\title{
Spatio-Temporal Rain Attenuation Model for Application to Fade Mitigation Techniques
}

\author{
Boris Christian Grémont, Member, IEEE, and Miodrag Filip, Member, IEEE
}

\begin{abstract}
We present a new stochastic-dynamic model useful for the planning and design of gigahertz satellite communications using fade mitigation techniques. It is a generalization of the Maseng-Bakken and targets dual-site dual-frequency rain attenuated satellite links. The outcome is a consistent and comprehensive model capable of yielding theoretical descriptions of: 1) long-term power spectral density of rain attenuation; 2) rain fade slope; 3) rain frequency scaling factor; 4) site diversity; and 5) fade duration statistics using a novel method based on Markov Chains. We also present a simple rain attenuation synthesizer matching the predictions of the theoretical model.
\end{abstract}

Index Terms-Diversity, dynamic, propagation, rain, statistics.

\section{INTRODUCTION}

$\mathbf{I}$ $\mathrm{N}$ THE CONTEXT of millimeter wave satellite communications, fade mitigation techniques (FMTs), refer to adaptive communication systems that try and compensate in real time for tropospheric attenuation effects affecting the slant path. The broad design objective is to design communication systems which try and maximize overall channel utilization while meeting specific quality of service requirements [1] and [2]. In order to control FMT techniques in real-time, it is necessary to have a precise knowledge of most dynamic and statistical characteristics of rain-induced attenuation which is the main source of channel impairment encountered at frequencies above $10 \mathrm{GHz},[3]$.

While first-order statistics of rain exceeded on an average climatic year allow to determine the achievable long-term quality of service (e.g., Throughput and BER availability, [4]), a natural extension is to consider a second correlated link also affected by rain. This provides: 1) possibility to study systems using site-diversity system as a FMT, [5] and 2) analysis of simultaneous up- and down-link attenuations of a satellite link through a transparent transponder [6].

Other characteristics which are usually referred to as secondorder or dynamic characteristics [7] are useful for the design of the control algorithm that is required to drive any particular FMT [8] and [9]. Fade slope statistics, [10], have applications for the short-term prediction of rain attenuation which is useful whenever the deployment of an FMT is not instantaneous but rather has a finite response time. Fade duration and interfade duration statistics, ([11] and [12]) are necessary to evaluate the

Manuscript received April 30, 2002; revised March 25, 2003. This work was supported by the U.K. Engineering and Physical Sciences Research Council (EPSRC) under Grant GR/L37342.

The authors are with the University of Portsmouth, Department of Electronic \& Computer Engineering, Anglesea Building, Portsmouth, PO1 3DJ, U.K. (e-mail: boris.gremont@port.ac.uk).

Digital Object Identifier 10.1109/TAP.2004.827501 long-term behavior of a rain fade countermeasure. This could for example be used to dimension the required window (buffer) size in layers of typical protocol stacks. It could also be useful for the study of adaptive FMT system relying on so-called timediversity, [13]. Knowledge of the frequency scaling factor of rain attenuation, [14], is necessary for systems where the actual measurement of the fades is made at a frequency different from that of the actual information-bearing signals.

When no experimental data is available, it may be required to use a software simulator of the satellite faded channel, [15] for characterizing the short-term behavior of an FMT. To be of real use, any proposed rain synthesizer should be accompanied with corresponding theoretical first- and second-order statistics, [16], so that actual delivery of a suitable quality of service (QoS) can be assessed on a long-term basis, [17]. For maximum portability and ease of estimation, the number of required input parameters should be kept to a minimum while still achieving meaningful predictions.

This paper presents a generalization of the stochastic-dynamic model first proposed by Maseng and Bakken (MB) in [18] which attempts to match all these criteria. In particular, the MB model is extended to two arbitrarily correlated satellite links at two different carrier frequencies. It is also inspired from the seminal works in [19]-[21] which to a lesser extent attempted to achieve very similar objectives.

This dual-frequency scenario, with particular assumptions on the space-time cross-correlation function of the rainfield, will be shown to provide a mathematically-consistent way of deriving expressions for: 1) of rain attenuation's power spectral density (PSD); 2) dual-location site diversity systems; 3) rain attenuation frequency scaling factor; 4) rain fade slope statistics; and 5) a new computational method based on Markov chains [22] is also applied for evaluating rain fade durations statistics and other related quantities.

\section{Model Definition}

There is broad agreement that point rainfall rate can be modeled as a lognormal variable, [23]. A logical extension is that the distribution of rainrate at two points on the horizontal plane is jointly lognormal [21] with probability density function (pdf) given by (1) at the bottom of the next page, where $R_{1}$ and $R_{2}$ are the point rainfall rates (millimeter/hour) for the two locations 1 and 2, respectively, of interest and $r$ is the cross-correlation factor between $R_{1}$ and $R_{2}$.

As a short-hand, we will denote this by

$$
f\left(R_{1}, R_{2}, r\right) \equiv \Lambda_{R_{1}, R_{2}}\left(m_{1}, \sigma_{1}, r, m_{2}, \sigma_{2}\right) .
$$


The pdf is obtained from measurements from two spatially separated rain gauges. The parameters $\left\{m_{i}, \sigma_{i}\right\}, i=1,2$ describe the marginal lognormal statistics of point rainfalls, at two locations 1 and 2 of interest. These four parameters are easily estimated from empirical or predicted distribution function of rainfall rate (see e.g., [24] for the fitting method). The marginal complementary cumulative distribution functions (ccdf) of $R_{i}$ take the lognormal form

$f\left(R_{i}\right)=\frac{1}{\sqrt{2 \pi} \sigma_{i} R_{i}} \exp \left[-\left(\frac{\ln R_{i}-m_{i}}{\sigma_{i}}\right)^{2}\right] \equiv \Lambda_{R_{i}}\left(m_{i}, \sigma_{i}\right)$.

The correlation factor $r$ of point rainfall rates and is given by (Appendix A)

$$
r=\frac{e^{r^{\prime} \sigma_{1} \sigma_{2}}-1}{\sqrt{e^{\sigma_{1}^{2}}-1} \sqrt{e^{\sigma_{2}^{2}}-1}}, r^{\prime} \in[0,1]
$$

The quantity $r^{\prime}$ denotes the yet undefined cross-correlation factor for the reduced variables, $u_{i} \equiv\left(\ln R_{i}-m_{i}\right) / \sigma_{i}$ which are Gaussian with zero-mean and unit-variance (see Appendix A). Considering only the horizontal plane, we may emphasize that $r$ and thus $r^{\prime}$ depend on the space-time properties of the rainfall rate field by writing $r^{\prime}=f(x, y, t)$. Clearly, when $\{x, y, t\}=\{0,0,0\}$, the two raingauges are co-located in space and time. In this case, the two-point rainfall rates are perfectly correlated and bearing in mind that $m_{1}=m_{2}$, $\sigma_{1}=\sigma_{2}$ we need that $r^{\prime}=f(0,0,0)=1$ and $r=1$. Similarly, when $r=0$, the two raingauges are infinitely separated in space-time which implies that $r^{\prime}=f(\infty, \infty, \infty)=0$ so that from $r=0$. Thus we conclude that $r^{\prime}=f(x, y, t)$ is a decreasing function of its argument $\{x, y, t\}$.

Rainfall patterns can be considered from two different perspectives. The Lagrangian viewpoint is obtained when the observer follows the moving rain storm/cell on its path over the ground. In this case, the storm has an observable direction and velocity of movement. The birth, development and then decay of the raincell will appear to the observer as a time pattern. If only one observer exists (one raingauge on a moving carriage), no conclusions can be drawn about the size of the spatial properties or spacing between rain cells. From the Eulerian point of view, the raingauge is immobile on the ground surface while the rainfall structure is passing above. In this perspective, observed time pattern of the rainfall contains combined effects of space structure, i.e., their distance and size, development and decay, and the velocity of storm movement. It is not possible to distinguish the effects of different velocity from different size of raincells. A small raincell moving slowly may produce the same time pattern as a large cell moving fast and the birth and decay cannot be distinguished from their movement.
Let us define the separation distance between our two raingauges as $\rho \equiv \sqrt{\left(\left|x_{1}-x_{2}\right|\right)^{2}+\left(\left|y_{1}-y_{2}\right|\right)^{2}}$. Then assuming isotropy of the rainfield $r^{\prime}$ may take the form

$$
r^{\prime}=\exp \left(-\frac{\rho}{L}-\beta_{2}|t|\right)
$$

where $L$ denotes the characteristic size of raincells and $\beta_{2}$ accounts for birth and decay of raincells. Introducing the average advection velocity, $\overline{\boldsymbol{V}}=\left[\bar{V}_{x}, \bar{V}_{y}\right] \mathrm{m} / \mathrm{s}$, we can apply Taylor's hypothesis, [25] to the spatial component giving $\rho \approx \overline{\boldsymbol{V}}|t|$. We can thus rewrite (5) as

$$
r^{\prime}=\exp (-\beta|t|) \approx \exp \left(\frac{\rho \bar{V}}{L}\right)
$$

where $\beta=\bar{V} / L+\beta_{2}$ is a compound time constant accounting for spatial properties and birth and decay. As in most cases, it may prove difficult to separate spatial from temporal effects which both play a role in setting the value of the correlation function (this would require rain radar maps), it may be expeditious to assume that $\beta \approx \overline{\boldsymbol{V}} / L$ where $L$ is slightly modified to also include birth and decay. This approximation is the last term in (6).

We will see later that the correlation function given by (4) to (6) is consistent with the assumptions of the MB model and that they imply point rainfall and indeed rain attenuation on a slant path have a first-order PSD as often verified in experimental propagation studies, [26].

Approximation (6) gives a simple and powerful way of modeling dual-point rainfall intensity as encountered in terrestrial and/or satellite communications over relatively small time scale and small geographical extent. The use of Taylor's hypothesis and parameter $\beta$ in (6) allows to express the joint pdf of rainrate (2) as either $f\left(R_{1}, R_{2}, r(\rho)\right)$ or $f\left(R_{1}, R_{2}, r(t)\right)$ depending on whether our interest is placed on spatial or temporal variations of the rainfield respectively. We will use this spatio-temporal duality extensively to calculate below different first- and second-order characteristics of rain attenuation.

To get a rough estimate of $\beta$, we may consider a mean storm velocity of $10 \mathrm{~m} / \mathrm{s}$ and a raincell mean diameter of $5 \mathrm{~km} \mathrm{[27].}$ Assuming isotropy, we get an estimate of $\beta \approx 10 / 5000=$ $2.0 \cdot 10^{-3}\left[\mathrm{~s}^{-1}\right]$. This compares favorable to the experimental values $1.68 \cdot 10^{-3}\left[\mathrm{~s}^{-1}\right]$ and $1.85 \cdot 10^{-3}\left[\mathrm{~s}^{-1}\right]$ identified in [18] and [28].

Defining $D_{i} \mathrm{~km}$ as the horizontal projections of the slant paths with elevation angles $\theta_{i}, H_{i}$ as local rain heights and $h_{i}$ as the altitudes of the stations, we can calculate the projection of the paths on the $x y$ plane with $D_{i}=\left(H_{i}-h_{i}\right) / \tan \theta_{i}$. Assuming homogeneity, the total path attenuation is $A_{i}=\Gamma_{i} \times$ $D_{i} \mathrm{~dB}$ where the specific attenuations are $\Gamma_{1}=a R_{1}^{b}, \Gamma_{2}=$

$$
\begin{aligned}
f\left(R_{1}, R_{2}, r\right)= & \frac{1}{2 \pi \sigma_{1} \sigma_{2} R_{1} R_{2} \sqrt{1-r^{2}}} \cdot \\
& \cdot \exp \left[-\frac{1}{2\left(1-r^{2}\right)}\left(\frac{\left(\ln R_{1}-m_{1}\right)^{2}}{\sigma_{1}^{2}}-\frac{2 r\left(\ln R_{1}-m_{1}\right)\left(\ln R_{2}-m_{2}\right)}{\sigma_{1} \sigma_{2}}+\frac{\left(\ln R_{2}-m_{2}\right)^{2}}{\sigma_{2}^{2}}\right)\right]
\end{aligned}
$$




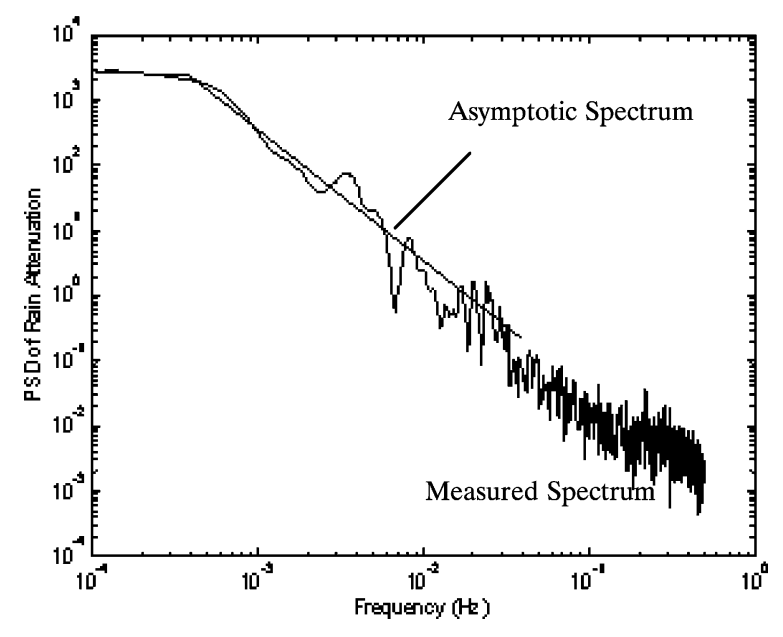

Fig. 1. Experimental PSD displaying a first-order characteristic.

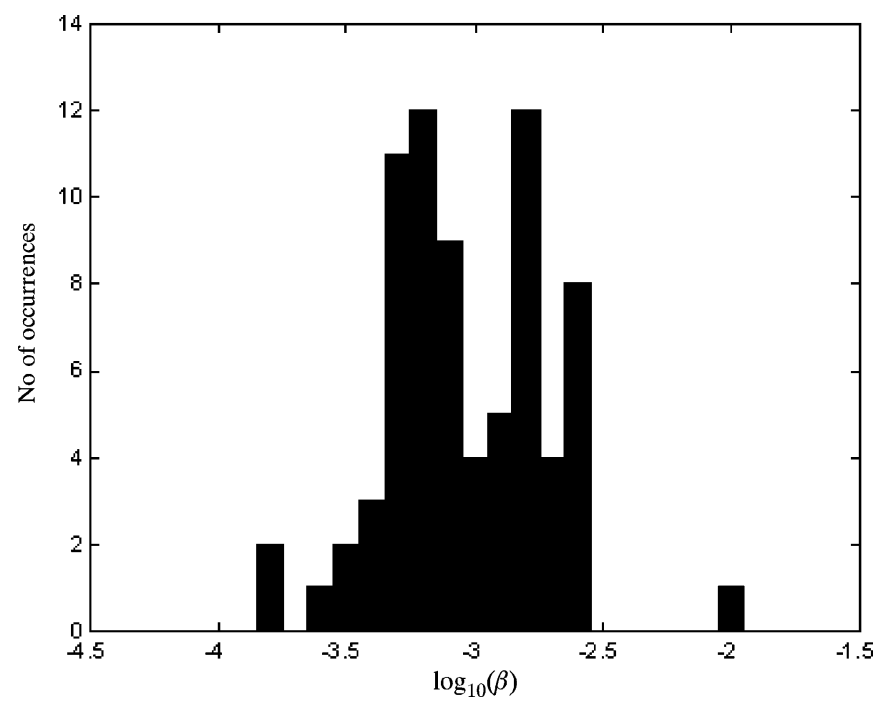

Fig. 2. Histogram of empirical values of parameter beta (Milan at $40 \mathrm{GHz}$ ).

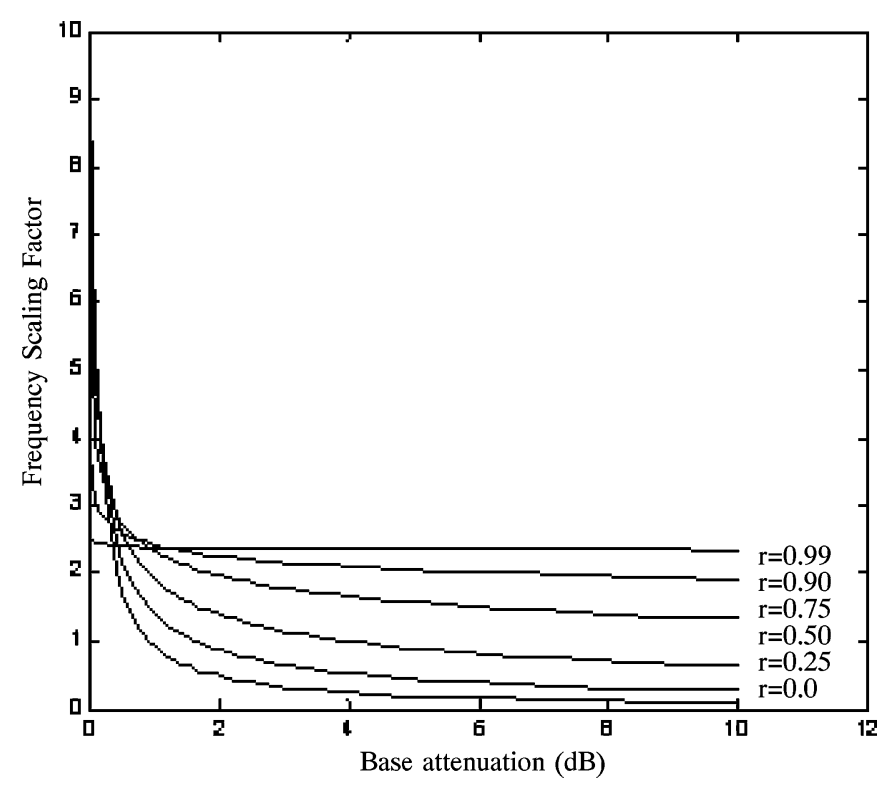

Fig. 3. Theoretical conditional frequency scaling factor for two links lying in ITU-R rainzone $\mathrm{K}$ at 39.12 and $20.1 \mathrm{GHz}$. $c R_{2}^{d}$. The use of the pairs $\{a, b\}$ and $\{c, d\}$ implies that we generally consider two different carrier frequencies and/or drop size distributions. Using a Jacobean technique described in [29], it is quite straight forward to prove that the distribution of joint rain attenuation is also lognormal with pdf

$$
f\left(A_{1}, A_{2}, r_{A}\right)=\Lambda_{A_{1}, A_{2}}\left(M_{1}, \Sigma_{1}, r_{A}, M_{2}, \Sigma_{2}\right)
$$

where:

$$
\left\{\begin{array}{l}
M_{1} \equiv b m_{1}+\ln (a)+\ln \left(D_{1}\right), \Sigma_{1}=b \sigma_{1} \\
M_{2} \equiv d m_{2}+\ln (c)+\ln \left(D_{2}\right), \Sigma_{2}=d \sigma_{2}
\end{array}\right.
$$

and

$$
r_{A}=\frac{e^{r^{\prime} \Sigma_{1} \Sigma_{2}}-1}{\sqrt{e^{\Sigma_{1}^{2}}-1} \sqrt{e^{\Sigma_{2}^{2}}-1}} .
$$

\section{DYNAMIC ChARACTERISTICS OF RAIN FADING}

\section{A. Spectrum of Rain Attenuation and Identification of $\beta$}

The work of Maseng and Bakken in [18] is extended in Appendix B to provide an exact solution for the long-term PSD, $P_{y y}(\omega)$ of rain attenuation which is shown to be

$$
P_{y y}(\omega)=2 e^{2 m} e^{\sigma^{2}} \sum_{n=1}^{\infty} \frac{\sigma^{2 n}}{n ! n \beta} \cdot \frac{1}{1+\left(\frac{\omega}{n \beta}\right)^{2}}+e^{2 m} e^{\sigma^{2}} \delta(\omega) .
$$

In practice, the first 20 terms in the summation are sufficient to converge to a good estimate of the PSD. Here and $m=M_{i}$ and $\sigma=\Sigma_{i}, i=1,2$ [see (8)]. A more useful approximation is the expected long-term asymptotic PSD of rain attenuation which is shown in Appendix B to be equal to

$$
\begin{aligned}
P_{y y}^{\infty}(\omega) & \approx \frac{2 \beta \sigma^{2} e^{2 m} e^{2 \sigma^{2}}}{\omega^{2}}, \omega>\omega_{c} \\
P_{y y}^{0}(\omega) & \approx 0.1745 \cdot \frac{e^{2 m} e^{\sigma^{2}} e^{2.648 \sigma}}{\beta}, 0<\omega \leq \omega_{c} \\
\omega_{c} & \approx 3.3855 \cdot \frac{\beta \sigma e^{\sigma^{2} / 2}}{e^{1.324 \sigma}} .
\end{aligned}
$$

This expression shows that the asymptotic PSD of rain attenuation has a first-order low-pass characteristic [30]. This indicates that the choice of cross-correlation function (6) is quite appropriate. Furthermore, the space-time parameter $\beta$ will affect the whole shape of the spectrum. In particular, for given marginal statistical parameters $(m, \sigma)$, a large value of $\beta$ will tend to lower the plateau in the PSD while the cutoff frequency of the spectrum will move toward the higher frequencies. Hence, second-order dynamic properties such as fade slope and fade durations will change significantly depending on the actual value of $\beta$. Note, that the cutoff $\omega_{c} \mathrm{rad} / \mathrm{s}$ is independent of the median value $e^{m}$ of rain attenuation. This gives an easy way to identify a value of the dynamic factor $\beta$ for particular events (or average PSD on a long-term basis). For this, one needs to compute the PSD of rain attenuation showing clearly the cutoff frequency. The variance of the event can be estimated for this particular event in the time domain from which $\beta$ can then calculated using the inverted form of the last equation in (11). 
TABLE I

ESTIMATED MEAN SCALING FACTORS COMPARED TO ITU-R'S

\begin{tabular}{c|c|c|c|c|c}
\hline $\begin{array}{c}\text { Frequency } \\
(\mathrm{GHz})\end{array}$ & 14 & 20 & 30 & 40 & 50 \\
\hline 12 & $1.36 / 1.34$ & $2.39 / 2.34$ & $4.35 / 4.27$ & $5.87 / 5.76$ & $6.89 / 6.76$ \\
\hline 14 & - & $1.72 / 1.73$ & $3.14 / 3.14$ & $4.24 / 4.24$ & $4.97 / 4.98$ \\
\hline 20 & - & - & $1.80 / 1.84$ & $2.43 / 2.48$ & $2.86 / 2.91$ \\
\hline 30 & - & - & - & $1.34 / 1.37$ & $1.58 / 1.61$ \\
\hline 40 & - & - & - & - & $1.17 / 1.20$ \\
\hline
\end{tabular}

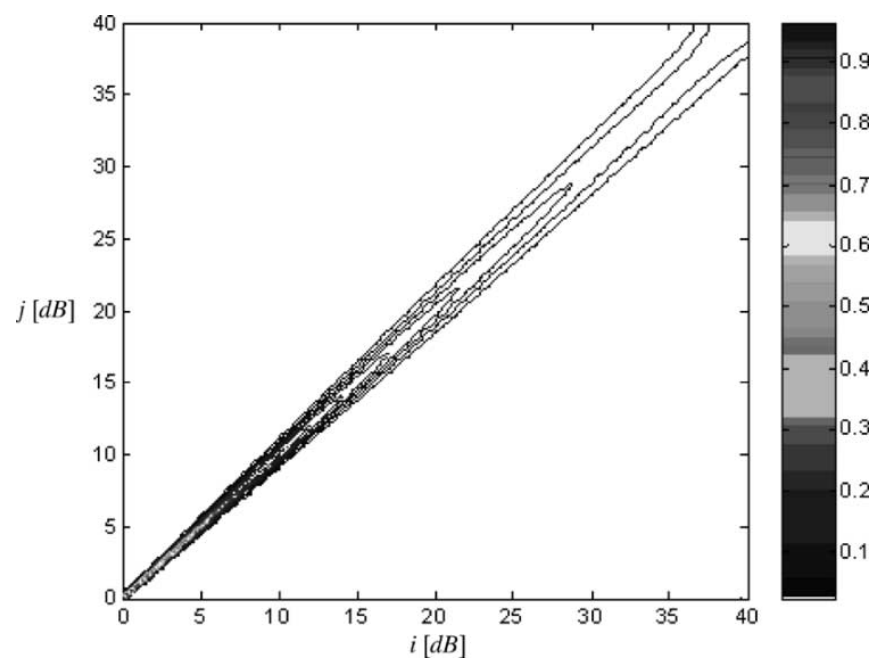

Fig. 4. Contour plot of the transition matrix.

This identification method has been applied to a particular event of about one hour from a received beacon signal data for southern England. The PSD is shown in Fig. 1. Note, that for high frequencies the PSD of rain attenuation tends to a $f^{-2}$ law in agreement with experimental results [26], [30]. The identified value of $\beta$ is about $4 \times 10^{-4}$. This is quite smaller than the long-term values reported in Section I. This may be due to a smaller advection velocity of the raincell or a larger raincell characteristic diameter. The identification of $\beta$ has been applied to around 70 rain events of ITALSAT beacon data collected in Milan over the year 1998. The histogram in Fig. 2 shows that $3.16 \times 10^{-4} \leq \beta \leq 3.16 \times 10^{-3}$. This demonstrates that for a particular location, $\beta$ varies over one order of magnitude. Furthermore, the central value is located at about $\beta \approx$ $7.9 \times 10^{-4} \mathrm{~s}^{-1}$ which is also smaller than the values reported in [18] and [28]. This indicates that the dynamic parameter is probably dependent on local climate. Thus, ultimately, maps of $\beta$ would be required for an accurate worldwide space-time rain model.

\section{B. Frequency Scaling Factor of Rain Attenuation}

Starting from (7), a new statistical model for the frequency scaling factor of rain attenuation is now developed. Let us consider the frequency scaling factor defined by [36]

$$
z \equiv \frac{A_{1}}{A_{2}}
$$

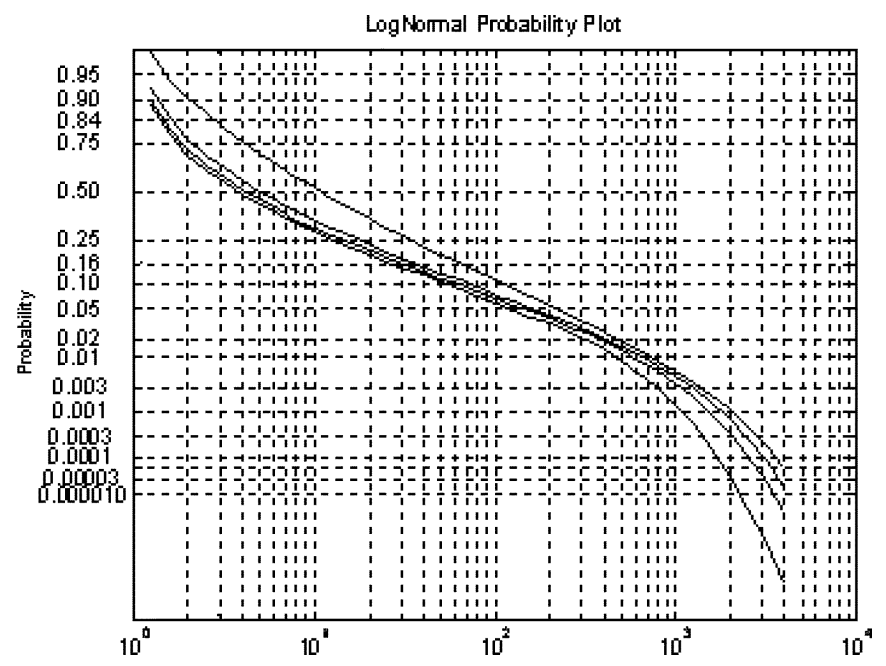

Fig. 5. Fade duration exceedance probability for attenuation thresholds of 1 (top), 3, 5, and 7 (bottom) dB.

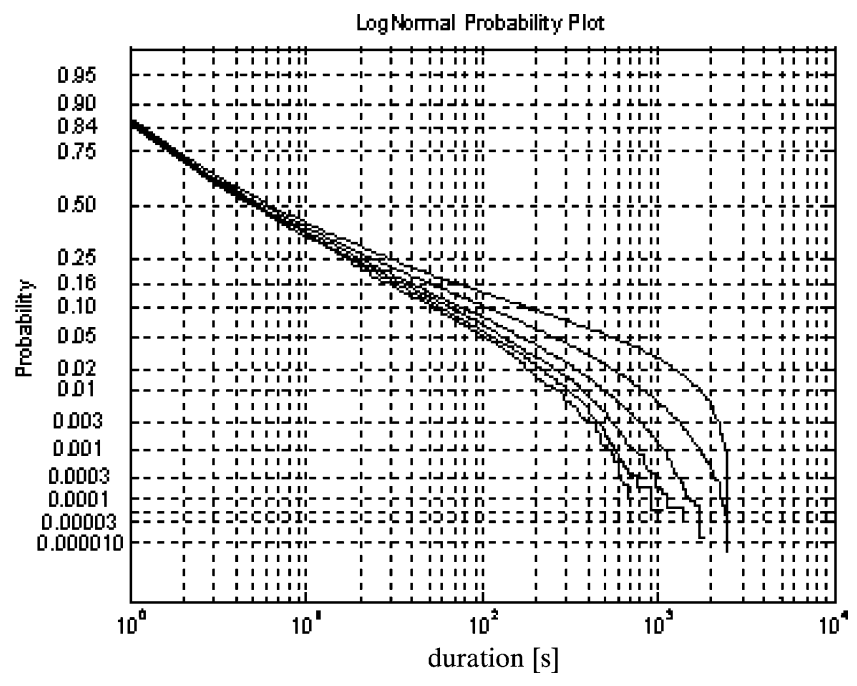

Fig. 6. Conditional Exceedance fade duration statistics for attenuation thresholds of 0.1 (top), 1, 3, 5, and 10 (bottom) dB from simulation.

Applying a Jacobean technique [29], it can easily be shown that the joint pdf of $\mathrm{z}$ and $A_{2}$ is

$$
\begin{aligned}
f\left(z, A_{2}\right) & =\left|A_{2}\right| \cdot f\left(z \cdot A_{2}, A_{2}, r_{A}\right) \\
& =A_{2} \cdot \Lambda_{z A_{2}, A_{2}}\left(M_{1}, \Sigma_{1}, r_{A}, M_{2}, \Sigma_{2}\right) .
\end{aligned}
$$

Then, the conditional pdf of the frequency scaling factor can be obtained from Bayes' theorem

$$
f\left(z \mid A_{2}\right)=\frac{f\left(z, A_{2}\right)}{f\left(A_{2}\right)}
$$

where [see (3)], $f\left(A_{2}\right)=\Lambda_{A_{2}}\left(M_{2}, \Sigma_{2}\right)$. 


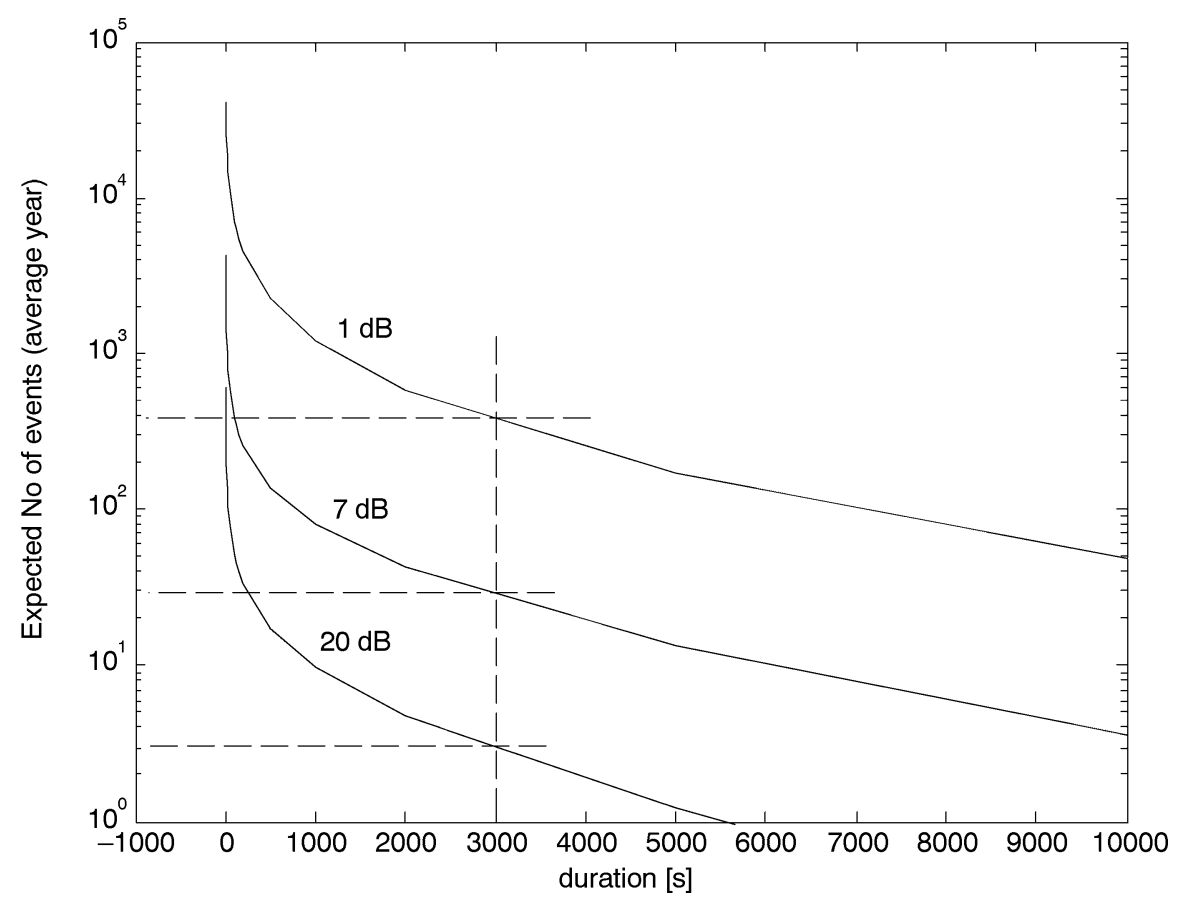

Fig. 7. Estimated number of events of a given duration.

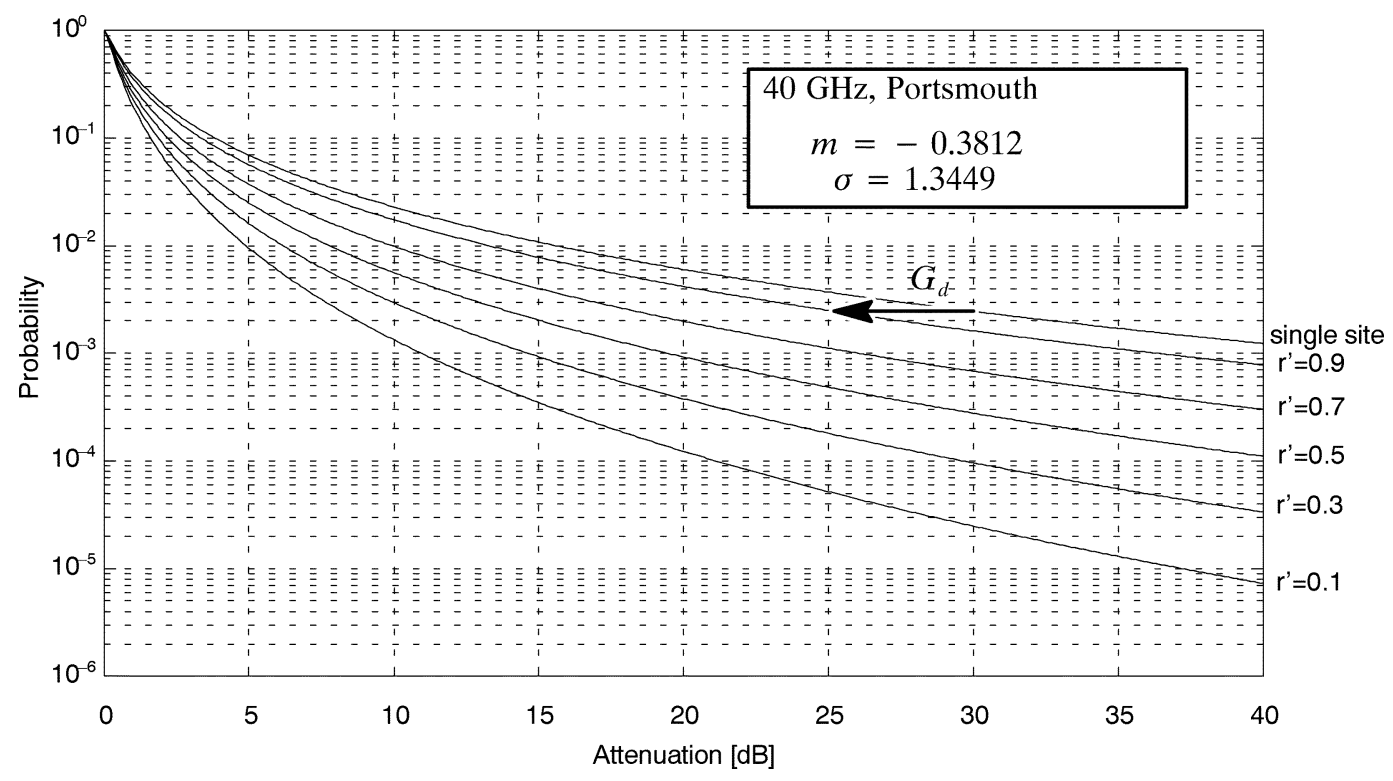

Fig. 8. Performance of dual-site single rainzone diversity system (Portsmouth at $40 \mathrm{GHz}$ ).

Of practical interest to FMTs is the conditional expected value of the frequency scaling factor given as a function of the base attenuation, i.e., the quantity $E\left\{z \mid A_{2}\right\}$ which can be easily be computed from (14) yielding

$$
E\left\{z \mid A_{2}\right\}=\int_{0}^{\infty} z \cdot f\left(z \mid A_{2}\right) \cdot d A_{2} .
$$

In expanded form (without algebraic simplifications), this is written as

$$
E\left\{z \mid A_{2}\right\}=\int_{0}^{\infty} z \times \frac{\exp \left[-\frac{\left(\mathcal{A}^{2}-2 r_{A} \mathcal{A} \mathfrak{B}+\mathfrak{B}^{2}\right)}{2\left(1-r_{A}^{2}\right)}\right]}{\frac{\sqrt{2 \pi}}{2 \pi \sqrt{1-r_{A}^{2}} \Sigma_{1} \Sigma_{2} z A_{2} \exp \left[-\frac{\mathfrak{B}^{2}}{2}\right]}} d z
$$

where $\mathcal{A} \equiv\left(\ln \left(z A_{2}\right)-M_{1}\right) / \Sigma_{1}$ and $\mathfrak{B} \equiv\left(\ln \left(A_{2}\right)-M_{2}\right) / \Sigma_{2}$.
Usually, the scaling factor is chosen so that it is greater than unity i.e., the carrier frequency corresponding to $A_{1}$ is greater than that corresponding to $A_{2}$. If instead, we choose the definition that $A_{1}<A_{2}$, then the integral (16) should be integrated over the interval $[0,1]$, which may offer a computational advantage. We have estimated the parameters, $\left\{M_{i}, \Sigma_{i}\right\}$ of the marginal lognormal pdfs for $A_{1}$ and $A_{2}$, so that a good fit is obtained with the ccdf predictions of the ITU-R rain attenuation model. We assumed that the carrier frequency for $A_{1}$ is $32.12 \mathrm{GHz}$ while that for $A_{2}$ is $20.1 \mathrm{GHz}$, both links being in rainzone $\mathrm{K}$ of the ITU-R model. Although this particular case is typical of a $K a$ band satellite communications scenario, it is important to note that the model (16) allows the scaling of attenuations from 
different rainzones with arbitrary correlation factor. We will not attempt, however, to justify the applicability of the proposed model in its widest generality.

Using (16), we have computed the conditional mean $E\left\{z \mid A_{2}\right\}$ of the frequency scaling factor as a function of the base attenuation level. The correlation factor $r_{A}$ is kept as a free parameter to see its impact on the frequency scaling factor. The results in Fig. 3 indicate that the correlation factor plays an important role on the actual value of the scaling factor. In particular, the mean conditional scaling factor can be assumed as constant for correlation factors close to unity while the scaling factor becomes strongly base-attenuation dependent for low correlation coefficients.

As in most satellite communication scenarios, the requirement is to frequency-scale attenuation on links which are geographically overlapping, the two attenuation time-series will be highly correlated, and from Fig. 3, the mean scaling factor can be considered as constant. Like the ITU-R model, the model proposed here shows that the scaling factor depends on the actual frequency pair chosen for $A_{1}$ and $A_{2}$. As an example, the average frequency scaling factor for different frequency pairs lying in rainzone $\mathrm{K}$ has been computed and is shown in Table I. Comparing this prediction with the ITU-R model, we see that the agreement is very good. However, unlike the ITU-R model, (16) indicates that our scaling factor will depend on the rainzone of the two links of interest since it depends on the actual marginal statistics of attenuation through the parameters $\left\{M_{i}, \Sigma_{i}\right\}$.

\section{Fade Duration Statistics Using a Markov Chain Approach}

Rain fading exhibits a significant degree of time correlation, so that the memory in the satellite environment cannot be neglected. For voice, outage probability is usually defined as the probability that the signal-to-noise ratio (SNR) is below a certain threshold. However, for packet-based data communications, the duration of a channel outage is also very important in determining its effect on data link and/or transport layer in the OSI protocol stack (or equivalent). More particularly, some error pattern, linked to long rain events, will result in unrecoverable or very serious errors which may lead to time-out expiration, congestion (or transmission) window shrinking or even connection shutdown. Within this context, a more appropriate definition of an outage event is that when the SNR goes below a certain threshold $\epsilon \mathrm{dB}$ and stays there for longer than a certain duration, $\tau \mathrm{s}$. Different quantities may be chosen to characterize the durations of rain attenuation events, [37]. Basic choices should include the unconditional and conditional pdfs of fade durations which are linked by $f_{d}(d)=f(d \mid \epsilon) f(\epsilon)$ where $f(\epsilon)=\Lambda_{\epsilon}\left(M_{i}, \Sigma_{i}\right)$, $i=1$, 2. Although this may only be an approximation, it is mentioned in [37] that such a formula allows to separate effects characterizing location-dependent effect [embodied by $f(\epsilon)$ ]

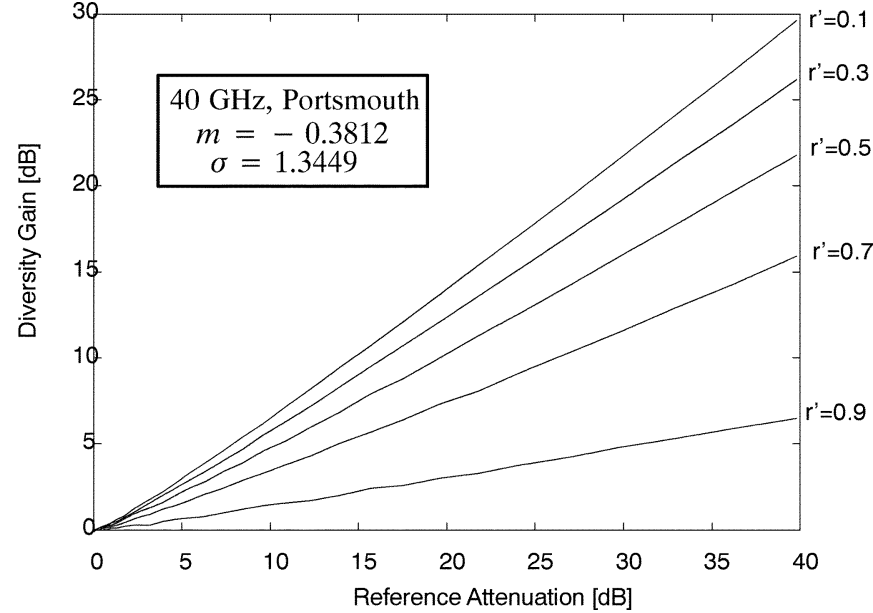

Fig. 9. Diversity gain as a function of reference attenuation (Portsmouth at $40 \mathrm{GHz}$ ).

from pure hydrometeor effect relating attenuation and duration via $f(d \mid \epsilon)$. A practical viewpoint is to determine the probability $\operatorname{Pr}\left\{d=\tau \mid A_{i} \geq \epsilon\right\}$ or the ccdf $\operatorname{Prob}\left\{d>\tau \mid A_{i} \geq \epsilon\right\}$.

Consider a discrete-time discrete amplitude Markov chain $X(n)$ representing the discretized version of rain attenuation. Assume a state space $\Omega=\{1,2, \ldots, N\} \mathrm{dB}$. Then the evolution of $X(n)$ can be completely described by the transition matrix $P$, whose elements are given by $P_{k l}=\operatorname{Prob}\{X(n+$ $\left.1)=l \mid X_{n}=k\right\}, k, l \in \Omega . P$ is such that for any of its rows (labeled by $k$ ) we have $\sum_{l} P_{k l}=1$ where $k$ is used to identify the columns of $\boldsymbol{P}$. The steady-state distribution linked to $\boldsymbol{P}$ will be denoted by the column vector $\pi$ and satisfies, assuming an arbitrary initial state, $\pi_{0} \pi=\lim \pi_{0} \boldsymbol{P}^{n}$ as $n \rightarrow \infty$. By setting $\Delta t=1 \mathrm{~s}, M_{1}=M_{2}=M, \Sigma_{1}=\Sigma_{2}=\Sigma$, and $A_{1}=k \mathrm{~dB}$ and $A_{2}=l \mathrm{~dB}$, we rewrite (7) as shown in (17) at the bottom of the page. The conditional pdf $f(l \mid k)$ can be found from Bayes' theorem yielding

$$
P_{k l}=\frac{\int_{k-0.5}^{k+0.5} \int_{l-0.5}^{l+0.5} f(k, l) d k d l}{\int_{k-0.5}^{k+0.5} \Lambda_{l}(m, \sigma) d l} .
$$

A typical output from (18) is shown in Fig. 4 for $M=-1.40$, $\Sigma=1.498$ and $\beta=1.65 \cdot 10^{-4}$. As most of the elements of $\boldsymbol{P}$ are equal to zero except around the diagonal $k=l$, the transitions from $k$ in $\mathrm{dB}$ to $l \mathrm{~dB}$ can only be small over one second. Now let $\epsilon \mathrm{dB}$ be an arbitrary attenuation threshold relevant to the FMT scenario being considered. We call $\mathfrak{B}$-states the bad states belonging to the attenuation subset $\mathfrak{B}=\{\epsilon+1, \ldots, N\}$ and $\mathcal{G}$-states the desired states belonging to $\mathcal{G}=\{1,2, \ldots, \epsilon\}$, [40]. The conditional exceedance probability of rain fade durations [37] can be computed using the machinery developed in [22]. For this, let us define a bad period as having a duration $T_{\mathfrak{B}} \equiv d[\mathrm{~s}]$ if a transition from a good state ( $\mathcal{G}$-state) to a bad

$$
\begin{aligned}
f(k, l) & =\frac{1}{2 \pi \Sigma^{2} k l \sqrt{1-r_{A}^{2}}} \exp \left[-\frac{1}{2\left(1-r_{A}^{2}\right)}\left(\frac{\left(\ln k-M^{2}\right)}{\Sigma^{2}}-\frac{2 r_{A}(\ln k-M)(\ln l-M)}{\Sigma^{2}}+\frac{\left(\ln l-M^{2}\right.}{\Sigma^{2}}\right)\right] \\
r_{A} & =\frac{\exp \left[\left(\sigma^{2} \exp [-\beta]-1\right)\right]}{\left(\exp \left[\sigma^{2}\right]-1\right)} .
\end{aligned}
$$


state, $\mathfrak{B}$-state, is followed by $d-1$ consecutive $\mathfrak{B}$-states, in turn followed by one $\mathcal{G}$-state. Zorzi showed that the duration statistics is

$$
\operatorname{Pr}\left\{T_{\mathfrak{B}} \geq d \mid A_{i} \geq \epsilon\right\}=\frac{\pi_{\mathcal{G}}^{T} \boldsymbol{P}_{\mathfrak{B}}^{d-1} \boldsymbol{P} \boldsymbol{e}_{\mathfrak{B}}}{\pi_{\mathcal{G}}^{T} \boldsymbol{P} \boldsymbol{e}_{\mathfrak{B}}}
$$

where $T$ denotes the transpose operator, $\pi_{\mathcal{G}}$ is obtained from the steady-state distribution by setting to zero all entries corresponding to states which are not in $\mathcal{G}$. $\boldsymbol{P}_{\mathfrak{B}}$ is obtained from $\boldsymbol{P}$ by setting to zero all $P_{k l}$ with $l \in \mathcal{G}$, and $\boldsymbol{e}_{\mathfrak{B}}$ is a column vector whose $k^{\text {th }}$ entry is one if $k \in \mathfrak{B}$.

Equation (19) is shown in Fig. 5 on a loglog plot. The graph shows that the exceedance probability of rain attenuation is lognormal in the range of durations between 10 and $1000 \mathrm{~s}$. The predicted shape and dependency on attenuation thresholds seems to fit quite well with ACTS results given in [37] but more detailed comparison to experimental results is needed.

The theoretical prediction has also been validated against simulated data using the rain synthesizer given in Appendix C. Clearly the agreement is good showing that Zorzi's method can be used to compute fade duration statistics.

We can also estimate the expected number of rain attenuation events greater than $\epsilon \mathrm{dB}$ with duration of $d \mathrm{~s}$ using

$$
N_{e}(d)=N_{s} \pi_{\mathcal{G}} \boldsymbol{P}_{\mathfrak{B}}^{d-1} \boldsymbol{P} \boldsymbol{e}_{\mathfrak{B}}
$$

where $N_{s}=31557600[\mathrm{~s}]$ is the number of seconds in the year.

We see in Fig. 7 that we can expect 400, 30, and 3 events of duration of $3000 \mathrm{~s}$ (50 mins) at the attenuation levels 1, 7, and $20 \mathrm{~dB}$, respectively. This is quite plausible at $50 \mathrm{GHz}$. Furthermore, as explained in [40], we note that the number of events for very short durations tends asymptotically to very large values. A great advantage is that the Markov chain model, thanks to its quantization, yields a finite number of events which is quite an advantage.

\section{Fade Slope Model}

The instantaneous rate of change of rain attenuation is commonly referred to as the fade slope. It is particularly relevant for systems employing predictive FMTs as investigated previously [33], [34]. Formally, the fade slope may be defined as follows:

$$
s(t)=\frac{y(t+\Delta t)-y(t)}{\Delta t} .
$$

Based on the definition (21), detailed expressions for first order and conditional statistics of rain fade slope were derived in [35]. These expressions will not be repeated here however it will be remarked that they are totally compliant with the generalized propagation model presented in this paper.

\section{DuAL-Location Site Diversity Model}

Site Diversity (SDV) consists in the selection of the least attenuated link between two Earth stations pointing toward a same satellite at any particular point in time, [41], [42]. In other words, SDV results in selecting the best signal according to

$$
A_{d}(t)=\min \left(A_{1}(t), A_{2}(t)\right) .
$$

The region $\mathfrak{D}$ in the $A_{1}, A_{2}$ plane, such that $\min \left(A_{1}(t), A_{2}(t)\right) \geq w$ is the set of points such that $A_{1}(t) \geq w$ or $A_{2}(t) \geq w$, [29]. From this, it is clear that the ccdf of the (balanced) pure selection site diversity system is given by

$$
\operatorname{Prob}\left\{A_{d} \geq w\right\}=\int_{w}^{\infty} \int_{w}^{\infty} f\left(A_{1}, A_{2}, r_{A}\right) d A_{1} d A_{2}
$$

where the joint pdf is specified in (7). Equation (23) can be calculated more easily after using a double change of variables shown in (24) at the bottom of the page, where the cross-correlation factor, $r^{\prime}$, is given in (5) to (6) depending on assumptions. This reduces the problem of computing two-dimensional Gaussian distributions which is very well documented [43], [44].

A typical output computed from (24) is shown in Fig. 8 where we have considered a balanced site diversity link at $40 \mathrm{GHz}$ located in the vicinity of Portsmouth assuming a fixed effective path length through rain of $3.5 \mathrm{~km}$. Generalization to unbalanced SDV is straight forward (it would require changing the values of the integration limits). The site diversity gain, $G_{D}$, is defined as the (equi-probability) difference between the path attenuation encountered on a reference link and that obtained on a (dual-site) diversity system [45], [46] i.e., (see arrow in Fig. 8)

$$
G_{D} \equiv A_{i}^{p \%}-A_{12}^{p \%}, i=1,2 .
$$

This is shown in Fig. 9 using the numerical outputs from (24). We have considered a single rain-zone dual-path system. Thus the diversity gain needs only be given with reference to the marginal statistics of one of the two sites. If we had dual rainzone site diversity, the diversity gain would in general be different depending on whether site No1 or site No2 is considered to be the reference ccdf. Fig. 9 also shows the impact of the correlation factor [see (24)]. Quite expectedly, high correlation factors only provide a small diversity gain.

Finally, as indicated in (6), the cross-correlation factor is simply an image of the impact of the separation between the two sites of the diversity system. Clearly increasing the separation distance will result in lower cross-correlation and therefore larger diversity gains. This is best displayed by considering (24) as a function of $r^{\prime}$ where $w$ simply becomes a parameter. Inverting (6) allows us to express (26) and thus (25) as a function of separation distance, $\bar{\rho}$, i.e., of

$$
\rho=\frac{\bar{v}}{\beta} \ln r^{\prime}=-5000 \ln r^{\prime}[\mathrm{m}]=5 \ln r^{\prime}[\mathrm{km}]
$$

$$
\operatorname{Prob}\left\{A_{d} \geq w\right\}=\int_{\left(\ln w-M_{1}\right) / \Sigma_{1}}^{\infty} \int_{\left(\ln w-M_{2}\right) / \Sigma_{2}}^{\infty} \frac{1}{2 \pi \sqrt{1-r^{\prime 2}}} \exp \left[-\frac{1}{2\left(1-r^{\prime 2}\right)}\left(x^{2}-2 r^{\prime} x y+y^{2}\right)\right] d x d y
$$




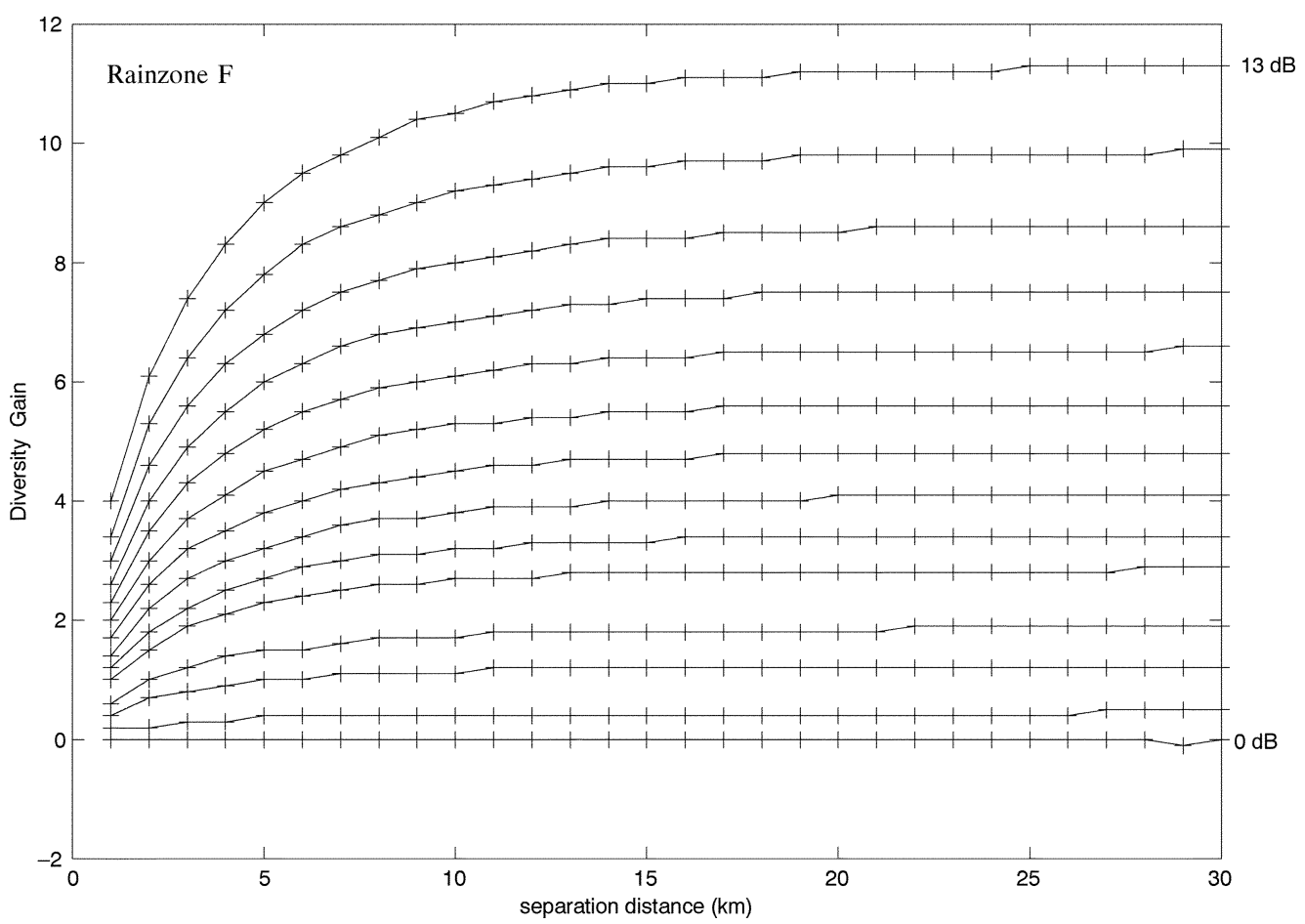

Fig. 10. Diversity gain as a function of separation distance for different values of reference attenuation (1 dB steps).

where we have used $\bar{v}=10 \mathrm{~m} / \mathrm{s}$ and $\beta=2 \cdot 10^{-3} \mathrm{~s}^{-1}$. This is shown in Fig. 10 for two site diversity links at 20 and $40 \mathrm{GHz}$ in the Portsmouth area. The improvement in diversity gain tends to stabilize for site separation above $20-25 \mathrm{~km}$. Clearly, this asymptotic behavior is scaled by the numerical value of $\bar{v} / \beta$ used in (27). Thus, the proposed model prompts us to estimate from experiment the average raincell velocity ([50]) as well as the value of the (long-term) spatio-temporal parameter $\beta$ at the reference location.

The diversity gain increases with higher levels of reference attenuation. This is consistent with the fact that high levels of rain fades are generally more localized and thus they are more likely to be unconnected between two separated links. Finally, the diversity gain shows a marked dependency on carrier frequency. This is consistent with the fact that fades of say $10 \mathrm{~dB}$ at $20 \mathrm{GHz}$ correspond to much more intense rainfall events and thus they are more localized compared to fades of $10 \mathrm{~dB}$ at say $40 \mathrm{GHz}$ which corresponds to a less intense and more widespread raincell. It is also important to note that the model proposed here concurs at least qualitatively with the predictions of other models for site diversity such as the Hodge model [45], [46]. However, unlike the Hodge model, the site diversity model derived here is not empirical and is totally coherent with the marginal cdfs for the rain attenuation encountered on our two slant paths.

Clearly, the use of a simple exponential spatial correlation function may not be accurate enough to model large scale site diversity. However it is believed that the model proposed in this paper is probably applicable to systems with maximum separation distances of up to 30 or $40 \mathrm{~km}$. If required, prediction inaccuracies can artificially be compensated for by selecting numerical values for input parameters $\beta$ and/or $\overline{\boldsymbol{V}}$ that minimize prediction errors when compared to empirical results (if available).

\section{CONCLUSION}

A generalized model of rain attenuation has been introduced. Particular emphasis is on deriving theoretical distributions for all quantities required for the design of communication systems using FMTs. This includes: 1) site diversity; 2) frequency scaling factor of rain attenuation; 3) fade slope statistics ([35]); 4) PSD of rain attenuation; and 5) computation of fade duration statistics using Markov Chains.

The proposed model is mathematically consistent and corresponds to a generalization of the Maseng-Bakken model to dual-frequency links. It relies on a particular exponential assumption for the space-time cross-correlation function of rainfield. Taylor's hypothesis is used to go from space to time (dynamic) characteristics. Although further validation is required, it is believed that the proposed generalized model is applicable to all propagation scenarios requiring statistical considerations of the dynamic characteristics of rain microwave fading and/or the modeling of site diversity systems typical of metropolitan areas $\left(60 \mathrm{~km}^{2}\right.$ or less).

The generalized model requires six main input parameters. The first classical four characterize the first order-lognormal statistics of rain attenuation They can easily be fitted to either experimental ccdfs or from global prediction models like the ITU-R's.

The last two parameters are new requirements imposed by the model proposed in this paper. The spatio-temporal parameter $(\beta)$ and the average velocity, $\bar{v}$, of raincells, are 
used to characterize the space-time properties of the rainfield at the location(s) of interest. $\beta$ was shown to vary over one order of magnitude for a particular satellite link and it is almost certain that it will also vary with actual location and climate. Similar expectations apply to the average speed of raincells. Thus maps of $\beta$ and $\bar{v}$ should ultimately be provided in addition to the standard ITU-R distributions for rainfall attenuation. A method to estimate $\beta$ in the frequency domain from classical experimental time-series has been presented. Preliminary work on estimation of local speeds of raincells is also underway for example in Portsmouth, [50].

\section{APPENDIX A}

\section{CORRELATION FunCTION OF RAIN ATtENUATION}

Let us consider a joint lognormal probability density function (pdf), $\mathcal{N}\left(0,1, r^{\prime}, 0,1\right)$, of two variables, $x$ and $y$, both with zero mean and unit variance and cross correlation given by $r^{\prime}$

$$
f(x, y)=\frac{1}{2 \pi \sqrt{1-r^{\prime 2}}} \exp \left[-\frac{1}{2\left(1-r^{\prime 2}\right)}\left(x^{2}-2 r^{\prime} x y+y^{2}\right)\right] .
$$

Now let $X=e^{\sigma_{x} x+m_{x}}$ and $Y=e^{\sigma_{y} y+m_{y}}$, then it is easily shown that $X$ and $Y$ are jointly lognormal with pdf (note the change of $r^{\prime}$ into $r$ ), see (A.2) shown at the bottom of the page, where $r$ is the cross-correlation factor between $X$ and $Y$ and is calculated below.

The (marginal) mean values of $X$ and $Y$ are

$$
\begin{aligned}
& E[X]=\exp \left(m_{x}+\frac{\sigma_{x}^{2}}{2}\right) \\
& E[Y]=\exp \left(m_{y}+\frac{\sigma_{y}^{2}}{2}\right)
\end{aligned}
$$

The standard deviations of $X$ and $Y$ are

$$
\begin{gathered}
\sigma_{X}=E\left[X^{2}\right]-E[X]=\exp \left(m_{x}+\frac{\sigma_{x}^{2}}{2}\right) \sqrt{\exp \left(\sigma_{x}^{2}\right)-1} \\
\sigma_{Y}=E\left[Y^{2}\right]-E[Y]=\exp \left(m_{y}+\frac{\sigma_{y}^{2}}{2}\right) \sqrt{\exp \left(\sigma_{y}^{2}\right)-1}
\end{gathered}
$$

The quantity $E[X Y]$, after applying the changes of variables $x=\left(\ln X-m_{x}\right) / \sigma_{x}$ and $y=\left(\ln X-m_{x}\right) / \sigma_{y}$ can be written as

$$
\begin{aligned}
& E[X Y]=e^{m_{x}} e^{m_{y}} \\
& \times \int_{-\infty}^{\infty} \int_{-\infty}^{\infty} \frac{e^{\sigma_{x}} e^{\sigma_{y}}}{2 \pi \sqrt{1-r^{\prime 2}}} \operatorname{Exp}\left[\frac{\left(x^{2}-2 r^{\prime} x y+y^{2}\right)}{2\left(1-r^{\prime 2}\right)}\right] d x d y
\end{aligned}
$$

The double integral in (A.7) can be shown to be equal to $\exp \left[\sigma_{x}^{2} / 2+\sigma_{y}^{2} / 2+r^{\prime} \sigma_{x} \sigma_{y}\right]$. Using equations (A.3)-(A.6), we can easily find the covariance function $C_{X Y}$

$C_{X Y}=E[X Y] E[X] E[Y]=e^{m_{x}+m_{y}} e^{\sigma_{x}^{2} / 2+\sigma_{y}^{2} / 2}\left(e^{r^{\prime} \sigma_{x} \sigma_{y}}-1\right)$

and the cross-correlation factor $r=r_{X Y}$ of the two lognormal variables is given by

$$
r=r_{X Y}=\frac{E[X Y]-E[X] E[Y]}{\sigma_{X} \sigma_{Y}}=\frac{e^{r^{\prime} \sigma_{x} \sigma_{y}}-1}{\sqrt{e^{\sigma_{x}^{2}}-1} \sqrt{e^{\sigma_{y}^{2}}-1}} .
$$

In the particular case where $m_{x}=m_{y} \equiv m$ and $\sigma_{x}=\sigma_{y} \equiv \sigma$, the covariance becomes

$$
C_{X X}=e^{2 m} e^{\sigma^{2}}\left(e^{r^{\prime} \sigma^{2}}-1\right)
$$

and the correlation function becomes

$$
r=r_{X Y}=\frac{e^{r^{\prime} \sigma^{2}}-1}{e^{\sigma^{2}}-1}
$$

\section{APPENDIX B}

\section{POWER Spectral Density of RAIN AtTenuation}

Assuming that $r^{\prime}=e^{-\beta|\tau|}$, the covariance function for the rain process, $y$, takes the form

$$
\begin{aligned}
C_{y y}(\tau) & =E[y(t) y(t+\tau)]-E[y(t)] E[y(t+\tau)] \\
& =e^{2 m} e^{\sigma^{2}}\left(e^{\sigma^{2} e^{-\beta|\tau|}}-1\right) .
\end{aligned}
$$

Using the Taylor series for $e^{\sigma^{2} Z}=\sum_{n=0}^{\infty} Z^{n} \sigma^{2 n} / n$ ! with $Z \equiv$ $e^{-\beta|\tau|}$, [47], (A.13) can be rewritten as

$$
\begin{aligned}
C_{y y}(\tau) & =e^{2 m} e^{\sigma^{2}}\left(\sum_{n=0}^{\infty} \frac{\sigma^{2 n} e^{-\beta n|\tau|}}{n !}-1\right) \\
& =e^{2 m} e^{\sigma^{2}}\left(\sum_{n=1}^{\infty} \frac{\sigma^{2 n} e^{-\beta n|\tau|}}{n !}-1\right)
\end{aligned}
$$

where we note that for $n=0$ the bracketed term in the middle of (A.14) is zero. Using the Fourier Transform pair:

$$
e^{-\beta n|\tau|} \leftrightarrow \frac{2 n \beta}{(n \beta)^{2}+\omega^{2}}=\frac{2}{n \beta} \cdot \frac{1}{1+\left(\frac{\omega}{n \beta}\right)^{2}}
$$

the PSD function of the rain process, $P_{y y}(\omega)$, is equal to the Fourier transform of its covariance function which gives

$$
P_{y y}(\omega)=2 e^{2 m} e^{\sigma^{2}} \sum_{n=1}^{\infty} \frac{\sigma^{2 n}}{n ! n \beta} \cdot \frac{1}{1+\left(\frac{\omega}{n \beta}\right)^{2}}+e^{2 m} e^{\sigma^{2}} \delta(\omega)
$$

where the Dirac function term represents the square of the mean value of the rain fading process. Although function (A.16) represents an exact solution and is easily computable (the summa-

$$
f(X, Y)=\frac{1}{2 \pi \sigma_{x} \sigma_{y} X Y \sqrt{1-r^{2}}} \exp \left[-\frac{1}{2\left(1-r^{2}\right)}\left(\frac{\left(\ln X-m_{x}\right)^{2}}{\sigma_{x}^{2}}-\frac{2 r\left(\ln X-m_{x}\right)\left(\ln Y-m_{y}\right)}{\sigma_{x} \sigma_{y}}+\frac{\left(\ln X-m_{x}\right)^{2}}{\sigma_{x}^{2}}\right)\right]
$$


tion is negligible for $n \gtrsim 20$ ), it is of practical interest to derive an asymptotic spectrum. In particular, if $(\omega / n \beta) \gg 1$, i.e., in the high frequency region, (A.16) is approximately equal to

$$
P_{y y}^{\infty}(\omega) \approx \frac{2 \beta e^{2 m} e^{\sigma^{2}}}{\omega^{2}} \cdot \sum_{k=1}^{\infty} \frac{n \sigma^{2 n}}{n !}=\frac{2 \beta \sigma^{2} e^{2 m} e^{2 \sigma^{2}}}{\omega^{2}}
$$

since $\sum_{k=1}^{\infty} n \sigma^{2 n} / n !=\sigma^{2} e^{\sigma^{2}}$.

Thus, the rain process displays a $f^{-2}$ characteristics in the high frequency region. In the case where $(\omega / n \beta) \ll 1$, i.e., in the low frequency region (but with $\omega \neq 0$ ), (A.16) becomes

$$
P_{y y}^{0}(\omega)=\frac{2 e^{2 m} e^{\sigma^{2}}}{\beta} \cdot \sum_{n=1}^{\infty} \frac{\sigma^{2 n}}{n ! n}
$$

The summation in (A.18) can be expressed in terms of the generalized hypergeometric function. However, a good approximation sufficient for our application is

$$
\sum_{n=1}^{\infty} \frac{\sigma^{2 n}}{n ! n} \approx e^{2.648 \sigma} e^{-2.439}, \quad 0.5 \leq \sigma \leq 2.0
$$

Thus

$$
P_{y y}^{0}(\omega) \approx 0.1745 \cdot \frac{e^{2 m} e^{\sigma^{2}} e^{2.648 \sigma}}{\beta} .
$$

The cutoff frequency $\omega_{c}$ of the spectrum can be determined by evaluating $\omega$ for which $P_{y y}^{0}(\omega)=P_{y y}^{\infty}(\omega)$. Using (A.18) and (A.20), it can be shown that

$$
\omega_{c}=3.3855 \cdot \frac{\beta \sigma e^{\sigma^{2} / 2}}{e^{1.324 \sigma}}
$$

\section{APPENDIX C SYNTHESIS OF TYPICAL RAIN EVENTS}

The MATLAB function, shown at the bottom of the page, generates lognormal time-series with a first-order spectral characteristics. This function corresponds to an implementation of the Maseng-Bakken model and it has been validated in terms of its first and second-order characteristics [48], [49]. This function has been used to determine simulated fade duration statistics (see Fig. 6).

\section{ACKNOWLEDGMENT}

The authors would like to thank Dr. C. Riva and Prof. A. Paraboni for provision of 1998 ITALSAT data within the framework of the COST280 programme. The authors would also like to thank the reviewers for their useful comments.

\section{REFERENCES}

[1] Inst. Elect. Eng., Inst. Elect. Eng. Colloquium Fade Countermeasures and Channel Restoration on Earth-Space Links London, U.K., Mar. 1990, vol. Digest no. 041.

[2] COST 225 Final Report, "Radiowave Propagation Modeling for New SatComm Services at Ku-Band and Above". COST 255. [Online]. Available: http://www.estec.esa.nl/xewww/cost255/index.html

[3] L. J. Ippolito, "Propagation effects and system performance considerations for satellite communications above $10 \mathrm{GHz}$," in Proc. IEEE Global Telecommunications Conf.: Connecting the Future GLOBECOM'90, vol. 1, Dec 2-5, 1990, pp. 89-91.

[4] B. C. Grémont, A. P. Gallois, M. Filip, and S. D. Bate, "Comparative analysis and performance of two predictive fade detection schemes for Ka band fade countermeasures," IEEE J. Select. Areas Commun., Special Issue on Direct-to-User Satellite Systems and Technologies at Ka Band and Beyond, vol. 17, pp. 180-192, Feb. 1999.

[5] J. Goldhirsh, B. H. Musiani, A. W. Dissanayake, and L. Kuan-Ting, "Three-site space-diversity experiment at $20 \mathrm{GHz}$ using ACTS in the Eastern United States," Proc. IEEE, vol. 85, pp. 970-980, June 1997.

[6] B. C. Gremont, M. Filip, and E. Vilar, "Evaluation of CNR statistics for satellite link with multiple countermeasures," Electron. Lett., vol. 36, no. 11, pp. 977-978, May 2000.

function $\mathrm{y}=\operatorname{synrain}\left(\mathrm{m} \_\right.$rain, sig_rain, duration_event)

\% Y_rain = synrain(m_rain, sig_rain, beta, duration)

$\%$ as a first order process

$\%$ m_rain, sig_rain: parameters of the lognormal pdf of rain

$\%$ duration_event is the No of samples to be generated ( 1 sample/sec)

\% Dr B. Gremont, University of Portsmouth, 1995

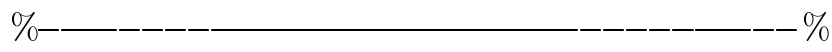

$\%$ Synthesize Rain $\quad \%$

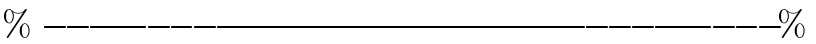

$\operatorname{randn}\left({ }^{\prime} \operatorname{seed}^{\prime}, \operatorname{sum}\left(100^{*}\right.\right.$ clock $\left.)\right)$;

$\mathrm{n} 1=\operatorname{randn}(1$, duration_event $)$;

$\mathrm{n} 1=(\mathrm{n} 1-\operatorname{mean}(\mathrm{n} 1)) \cdot / \operatorname{std}(\mathrm{n} 1) ;$

$[\mathrm{b}, \mathrm{a}]=\operatorname{BUTTER}(1$, beta. $/ 0.5)$;

$\mathrm{Y}=$ filter$(\mathrm{b}, \mathrm{a}, \mathrm{n} 1)$;

$\mathrm{Y}=(\mathrm{Y}-\operatorname{mean}(\mathrm{Y})) \cdot / \operatorname{std}(\mathrm{Y}) ; \quad \%$ normalize to zero mean and unit variance

$\mathrm{y} 2=\exp \left(\mathrm{m} \_\right.$rain $+\mathrm{Y} .{ }^{*}$ sig_rain $)$; 
[7] D. G. Sweeney and C. W. Bostian, "The dynamics of rain-induced fades," IEEE Trans. Antennas Propagat., vol. 40, pp. 275-278, Mar. 1992.

[8] G. Tartara, "Fade countermeasures in millimeter-wave satellite communications: A survey of methods and problems," in Proc. Olympus Utilization Conf., Vienna, Apr. 12-14, 1989, ESA SP-292, pp. 103-107.

[9] M. J. Willis and B. G. Evans, "Fade countermeasures at Ka band for Olympus," Int. J. Satellite Communicat., vol. 6, pp. 301-311, 1988.

[10] B. Nelson and W. L. Stutzman, "Fade slope on 10 to $30 \mathrm{GHz}$ earthspace communication links-measurements and modeling," Proc. Inst. Elect. Eng. Microwaves, Antennas and Propagation, vol. 143, no. 4, pp. 353-357, Aug. 1996.

[11] A. H. Cox and H. Hoffman, "Fade duration and interfade interval statistics measured on a 19-GHz earth-space path," IEEE Trans. Commun., vol. 30, pp. 265-269, Jan. 1982.

[12] A. Safaai-Jazi and W. L. Stutzman, "Empirical models for rain fade time on Ku- and Ka-band satellite links," IEEE Trans. Antennas Propagat., vol. 43, pp. 1411-1415, Dec. 1995.

[13] A. F. Ismail and P. A. Watson, "Characteristics of fading and fade countermeasures on a satellite-earth link operating in an equatorial climate, with reference to broadcast applications," Proc Inst. Elect. Eng. Microwaves, Antennas and Propagation, vol. 147, no. 5, pp. 369-373, Oct. 2000.

[14] D. Hodge, "Frequency scaling of rain attenuation," IEEE Trans. Antennas Propagat., vol. 25, pp. 446-447, May 1977.

[15] B. C. Grémont, "Software simulation of the satellite fading channel (UK EPSRC Grant GR/L37342)," Univ. Portsmouth, Microwave Telecommunication Systems Group, Internal Report No 98/2, 1998.

[16] Proc. OPEX 2nd Workshop of the Olympus Propagation Experimenters, Vol. 1: Reference Book on Attenuation Measurement and Prediction", Noordwijk, Nov. 8-10, 1994, WPP-083.

[17] M. H. Hadjitheodosiou, A. Ephremides, and D. Friedman, "Broadband Access Via Satellite,", CSHCN Tech Research Rep. 99-2 (ISR T.R. 99-9), 1999.

[18] T. Maseng and P. M. Bakken, "A stochastic model for rain attenuation," IEEE Trans. Commun., vol. COM-29, pp. 660-669, May 1981.

[19] R. M. Manning, "A unified statistical rain-attenuation model for communication link fade predictions and optimal stochastic fade control design using a location dependent rain-statistics database," Int. J. Satellite Commun., vol. 8, pp. 11-30, 1990.

[20] E. Matricciani, "Prediction of site diversity performance in satellite communications systems affected by rain attenuation: Extension of the twolayer model," Euro. Trans. Telecommun. Related Techno., vol. 5, no. 3 , May-June 1994

[21] R. K. Crane, Electromagnetic Wave Propagation through Rain. New York: Wiley, 1996.

[22] M. Zorzi, "Outage and error events in bursty channels," IEEE Trans. Commun., vol. 46, pp. 349-356, Mar. 1998

[23] S. H. Lin, "Statistical behavior of rain attenuation," Bell Systems Tech. J., vol. 52, no. 4, pp. 557-581, Apr. 1973.

[24] M. Filip and E. Vilar, "Optimum utilization of the channel capacity of a satellite link in the presence of amplitude scintillations and rain attenuation," IEEE Trans. Commun., vol. 38, pp. 1958-1965, Nov. 1990.

[25] G. I. Taylor, "Statistical theory of turbulence," in Proc. R. Soc., vol A164, London, U.K., 1938, pp. 476-490.

[26] E. Matricciani, "Physical-mathematical model of the dynamics of rain attenuation with application to power spectrum," IEE Electron. Lett., vol. 30, pp. 522-524, 1994.

[27] J. B. Valdes and I. Rodriguez-Iturbe, "Approximations of temporal rainfall from a multi-dimensional model," Water Resources Res., vol. 21, no. 8, pp. 1259-1270, Aug. 1985.

[28] A. Burgueño, E. Vilar, and M. Puigcerver, "Spectral analysis of rainfall rate and relation to fade dynamics," IEEE Trans. Commun., vol. 38, no. 9, pp. 1359-1366, Sept. 1990.

[29] A. Papoulis, Probability, Random Variables and Stochastic Processes: McGraw-Hill, 1991.

[30] Y. Karasawa and T. Matsudo, "Characteristics of fading on low-elevation angle earth-space paths with concurrent rain attenuation and scintillation," IEEE Trans. Commun., vol. 39, pp. 657-661, 1991.

[31] S. H. Lin, H. J. Bergmann, and M. V. Pursley, "Rain attenuation on earthsatellite paths -Summary of 10-year experiments and studies," Bell Syst. Tech. J., vol. 59, no. 2, p. 183, Feb. 1980.

[32] “ITU-R's "Rec. 838: Specific Attenuation Model for Rain Attenuation for Rain for Use in Prediction Methods," ITU, 2001.
[33] F. Rücker, "Frequency and attenuation dependent fade slope statistics," IEE Electron. Lett., vol. 29, no. 9, pp. 744-6, Apr. 1993.

[34] K. J. Timothy, J. T. Ong, and E. B. L. Choo, "Descriptive fade slope statistics on INTELSAT Ku-band communication link," IEE Electron. Lett., vol. 36, no. 16, pp. 1422-1424, Aug. 2000.

[35] B. C. Grémont and D. L. Ndzi, "First order and conditional statistics of rain attenuation fade slope," IEE Electron. Lett., vol. 36, no. 20, pp. 1733-1734, Sept. 2000.

[36] J. D. Laster and W. L. Stutzman, "Frequency scaling of rain attenuation for satellite communication links," IEEE Trans. Antennas Propagat., vol. 43, pp. 1207-1216, Nov. 1995.

[37] H. Helmken, R. E. Henning, J. Feil, L. J. Ippolito, and C. E. Mayer, "A three-site comparison of fade-duration measurements," Proc. IEEE, vol. 85, pp. 917-924, June 1997.

[38] E. Matricciani, M. Mauri, and A. Paraboni, "Dynamic characteristics of rain attenuation: Duration and rate of change," Alta Frequenza, vol. LVI, no. 1-2, pp. 33-45, Jan.-Apr. 1987.

[39] E. Vilar and A. Burgueño, "Analysis and modeling of time intervals between rain rate exceedances in the context of fade dynamics," IEEE Trans. Commun., vol. 39, pp. 1306-1312, Sept. 1999.

[40] A. Paraboni and C. Riva, "A new method for the prediction of fade duration statistics in satellite links above $10 \mathrm{GHz}$," Int. J. Satellite Commun., vol. 12, pp. 387-394, 1994

[41] L. J. Ippolito, Radiowave Propagation in Satellite Communications. New York: Van Nostrand Reinhold, 1986.

[42] A. Bosisio, C. Capsoni, and A. Paraboni, "Physical Modeling of Site Diversity and its Application to 20/30 GHz Earth Stations," ESA, ESA STM-255, 1995.

[43] M. Abramowitz and I. A. Stegun, Handbook of Mathematical Functions. New York: Dover, 1964.

[44] A. Genz. (2002) Numerical Computation of Bivariate and Trivariate Normal Probabilities. [Online]. Available: /http://www.sci.wsu.edu/ math/faculty/genz/papers/bvnn/bvnn.html

[45] D. B. Hodge, "An empirical relationship for path diversity gain," IEEE Trans. Antennas Propagat., vol. AP-24, pp. 250-251, Mar. 1976.

[46] _ , "An improved model for diversity gain on earth-space propagation paths," Radio Sci., vol. 17, no. 6, pp. 1393-1399, Nov.-Dec. 1982.

[47] C. Catalan, "An investigation of simultaneous fade dynamics and radiometry in a $40 \mathrm{GHz}$ satellite down-link," Ph.D. dissertation, Univ. Portsmouth, 1999.

[48] B. C. Grémont and M. Filip, "Simulation of a high frequency satellite link with a fade countermeasure," in Proc. Inst. Elect. Eng. Conf. Antennas Propagation, York, Mar./Apr. 1999, IEE Conf. Publ. No 461, pp. $164-168$.

[49] B. Audoire, L. Castanet, F. Christophe, and B. Grémont, "Ka band time-series generator and comparison with Olympus data," in Proc. 19th AIAA Int. Communications Satellite Systems Conf., Toulouse, France, Apr. 17-20, 2001.

[50] E. E. Vilar and F. P. Fontan, "Micro scale rain variations: Application to correlation," in Proc. U.K. Nat. URSI Symp., Malvern, U.K., Dec. 11-12, 2001

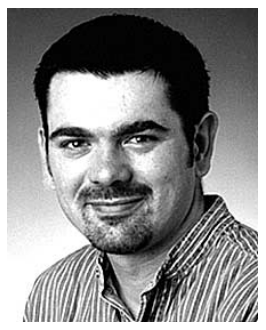

Boris Christian Grémont (M'96) was born in France on January 9, 1969. He received the DUT from the Universitary Institute of Technology (IUT), Grenoble, France, in 1992 and the Bachelor's degree (First Class Honors) in engineering and the Ph.D. degree in electrical electronic engineering from Coventry University, Coventry, U.K., in 1994 and 1998 , respectively.

After postdoctoral research at the Microwave Telecommunication Systems Research Group (MTSRG), Univerisity of Portsmouth, Portsmouth, U.K., he was appointed Senior Lecturer in the Department of Electrical Electronic Engineering (now the Electronics \& Computer Engineering Department). Currently, he teaches mathematics, communication systems, and data communnications \& networks at undergraduate and graduate levels. $\mathrm{He}$ is also involved in the European COST 280 action "Propagation Impairment Mitigation for Millimeter Wave Radio Systems" and he also contributes to the U.K.'s RAINMAP project (Fractal Rain Modeling for Millimeter Wave Propagation). His current research interests include earth-space, terrestrial and wideband propagation, satellite communications, DSP, and FMTs at physical, data link, and MAC layers. 


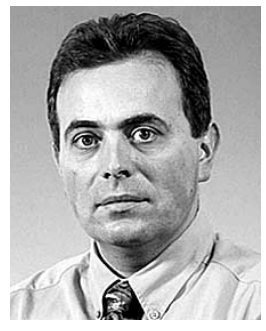

Miodrag Filip (S'88-M'91) was born in Belgrade, Yugoslavia, on April 21, 1963. He received the electronic engineering degree from the University of Belgrade, Belgrade, Yugoslavia, in 1987.

$\mathrm{He}$ was a Research Assistant for CAD in telecommunications at the Faculty of Electrical Engineering, University of Belgrade, from 1987 to 1988 , where he was involved in the design and planning of a space- and frequency-adaptive slow frequency hopping digital cellular radio-telephone network. In July 1988, he joined the Microwave Telecommunications and Signal Processing Research Group, Univerisity of Portsmouth, Portsmouth, U.K., where he completed Ph.D. research in advanced signal processing applied to fade countermeasures for low data rate digital VSAT communication systems at 20/30 GHz. From 1990 to 1993, he worked as an Electronics Design Engineer for De La Rue Systems Ltd., Havant, U.K., on a range of document-handling machines. He was appointed Senior Lecturer in the Department of Electrical and Electronic Engineering, University of Portsmouth, in 1993, and is now Principal Lecturer with teaching, consultancy, and research responsibilities in telecommunications, CAD, and microprocessor systems. Since 2001, he has been the Chairman of European COST Action 280 "Propagation Impairment Mitigation for mm-wave Radio Systems." His particular research interests are in VSAT satellite networks with fade countermeasures. 